\title{
EXISTENCE PRINCIPLES FOR SECOND ORDER NONRESONANT BOUNDARY VALUE PROBLEMS
}

\author{
DONAL O'REGAN \\ University College Galway \\ Department of Mathematics \\ Galway, IRELAND
}

(Received December, 1993; Revised April, 1994)

\begin{abstract}
We discuss the two point singular "nonresonant" boundary value problem $\frac{1}{p}\left(p y^{\prime}\right)^{\prime}=f\left(t, y, p y^{\prime}\right)$ a.e. on $[0,1]$ with $y$ satisfying Sturm Liouville, Neumann, Periodic or Bohr boundary conditions. Here $f$ is an $L^{1}$-Carathéodory function and $p \in C[0,1] \cap C^{1}(0,1)$ with $p>0$ on $(0,1)$.
\end{abstract}

Key words: Existence, Singular, Nonresonant, Boundary Value Problems, Sturm Liouville Problems.

AMS (MOS) subject classifications:34B15.

\section{Introduction}

In this paper, problems of the form

$$
\frac{1}{p(t)}\left(p(t) y^{\prime}(t)\right)^{\prime}=f\left(t, y(t), p(t) y^{\prime}(t)\right) \text { a.e. on }[0,1]
$$

are discussed with $y$ satisfying either

(i) (Sturm Liouville)

$$
\left\{\begin{array}{l}
-\alpha y(0)+\lim _{t \rightarrow 0^{+}} p(t) y^{\prime}(t)=c_{0}, \alpha \geq 0, \beta \geq 0, \alpha^{2}+\beta^{2}>0 \\
a y(1)+b_{t \rightarrow 1_{-}} p(t) y^{\prime}(t)=c_{1}, a \geq 0, b \geq 0, a^{2}+b^{2}>0 \\
\max \{a, \alpha\}>0
\end{array}\right.
$$

(ii) (Neumann)

$$
\left\{\begin{array}{l}
\lim _{t \rightarrow 0^{+}} p(t) y^{\prime}(t)=c_{0} \\
\lim _{t \rightarrow 1_{-}} p(t) y^{\prime}(t)=c_{1}
\end{array}\right.
$$


(iii) (Periodic)

or

$$
\left\{\begin{array}{l}
y(0)=y(1) \\
\lim _{t \rightarrow 0^{+}} p(t) y^{\prime}(t)=\lim _{t \rightarrow 1_{-}} p(t) y^{\prime}(t)
\end{array}\right.
$$

(iv) (Bohr)

$$
\left\{\begin{array}{l}
y(0)=c_{0} \\
\int_{0}^{1} \frac{d s}{p(s)} \lim _{t \rightarrow 1} p(t) y^{\prime}(t)-y(1)=c_{1} .
\end{array}\right.
$$

Remark: If a function $u \in C[0,1] \cap C^{1}(0,1)$ with $p u^{\prime} \in C[0,1]$ satisfies boundary condition $(\underline{i})$, we write $u \in(S L)$. A similar remark applies for the other boundary condition. If $u$ satisfies $(\underline{i})$ with $c_{0}=c_{1}=0$, we write $u \in(S L)_{0}$, etc.

Throughout the paper, $p \in C[0,1] \cap C^{1}(0,1)$ together with $p>0$ on $(0,1)$. Also $p f:[0,1] \times$ $\mathbf{R}^{2} \rightarrow \mathbf{R}$ is an $L^{1}$-Carathéodory function. By this we mean:

(i) $\quad t \rightarrow p(t) f(t, y, q)$ is measurable for all $(y, q) \in \mathbf{R}^{2}$,

(ii) $\quad(y, q) \rightarrow p(t) f(t, y, q)$ is continuous for a.e. $t \in[0,1]$,

(iii) for any $r>0$ there exists $h_{r} \in L^{1}[0,1]$ such that $|p(t) f(t, y, q)| \leq h_{r}(t)$ for a.e. $t \in[0,1]$ and for all $|y| \leq r,|q| \leq r$.

The results in the literature $[7,10,13-16]$ concern the nonresonant second order problem

$$
\left\{\begin{array}{l}
y^{\prime \prime}+f(t, y)=0 \text { a.e. on }[0,1] \\
y \in(S L),(N) \text { or }(P)
\end{array}\right.
$$

In particular, if $\frac{f(t, y)}{y}$ stays asymptotically between two consecutive eigenvalues or to the left of the spectrum of the differential operator then certain existence results can be established. The most advanced results to date seem to be [7], where quadratic forms associated with the eigenvalues and eigenfunctions are used to establish various existence criteria.

This paper deals with the more general problem (1.1). By using properties of the Green's function and by examining appropriate Sturm Liouville eigenvalue problems, we are able to establish various existence results. The paper will be divided into three sections. In section 2, fixed point methods, in particular a nonlinear alternative of Leray-Schauder type, will be used to establish existence principles for (1.1) with the various boundary conditions. We remark here that the existence principles are constructed with the nonresonant problem in mind. Section 3 establishes various existence theorems and section 4 discusses the Sturm Liouville eigenvalue problem.

In the remainder of the introduction we gather together some facts on second order differential equations which will be used throughout this paper. For notational purposes, let $w$ be a weight function. By $L_{w}^{1}[0,1]$ we mean the space of functions $u$ such that $\int_{0}^{1} w(t)|u(t)| d t<\infty$. $L_{w}^{2}[0,1]$ denotes the space of functions $u$ such that $\int_{0}^{1} w(t)|u(t)|^{2} d t<\infty$; also for $u, v \in L_{w}^{2}[0,1]$ define $\langle u, v\rangle=\int_{0}^{1} w(t) u(t) \overline{v(t)} d t$. Let $A C[0,1]$ be the space of functions which are absolutely continuous on $[0,1]$. 
Theorem 1.1: Suppose

and

$$
p \in C[0,1] \cap C^{1}(0,1) \text { with } p>0 \text { on }(0,1) \text { and } \int_{0}^{1} \frac{d s}{p(s)}<\infty
$$

$$
r, g \in L_{p}^{1}[0,1]
$$

are satisfied. Then

$$
\left\{\begin{array}{l}
\frac{1}{p}\left(p y^{\prime}\right)^{\prime}+r(t) y=g(t) \text { a.e. on }[0,1] \\
y(0)=a_{0}, \lim _{t \rightarrow 0^{+}} p(t) y^{\prime}(t)=b_{0}
\end{array}\right.
$$

has exactly one solution $y \in C[0,1] \cap C^{1}(0,1)$ with $p y^{\prime} \in A C[0,1]$. (By a solution to (1.5), we mean a function $y \in C[0,1] \cap C^{1}(0,1), p y^{\prime} \in A C[0,1]$ which satisfies the differential equation a.e. on $[0,1]$ and the stated initial condition).

Proof: Let $C[0,1]$ denote the Banach space of continuous functions on $[0,1]$ with norm

$$
|u|_{K}=\sup _{t \in[0,1]}\left|e^{-K R(t)} u(t)\right| \text { where } K=\int_{0}^{1} \frac{d s}{p(s)} \text { and } R(t)=\int_{0}^{t} p(s) r(s) d s .
$$

Solving (1.5) is equivalent to finding a $y \in C[0,1]$ which satisfies

$$
y(t)=a_{0}+b_{0} \int_{0}^{t} \frac{d s}{p(s)}+\int_{0}^{t} \frac{1}{p(s)} \int_{0}^{s} p(s)[-r(x) y(x)+g(x)] d x d s .
$$

Define the operator $N: C[0,1] \rightarrow C[0,1]$ by

Now $N$ is a contraction since

$$
N y(t)=a_{0}+b_{0} \int_{0}^{t} \frac{d s}{p(s)}+\int_{0}^{t} \frac{1}{p(s)} \int_{0}^{s} p(x)[-r(x) y(x)+g(x)] d x d s .
$$

$$
\begin{aligned}
& |N u-N v|_{K} \leq|u-v|_{K} \max _{t \in[0,1]}\left|e^{-K R(t)} \int_{0}^{t} \frac{1}{p(s)} \int_{0}^{s} p(x) r(x) e^{K R(x)} d x d s\right| \\
= & \frac{|u-v|_{K}}{K} \max _{t \in[0,1]}\left|e^{-K R(t)} \int_{0}^{t} \frac{1}{p(s)}\left[e^{K R(s)}-1\right] d s\right| \leq|u-v|_{K}\left[1-e^{-K R(1)}\right] .
\end{aligned}
$$

The Banach contraction principle now establishes the result.

Let $u_{1}$ be the unique solution to

$$
\left\{\begin{array}{l}
\frac{1}{p}\left(p y^{\prime}\right)^{\prime}+r(t) y=0 \text { a.e. on }[0,1] \\
y(0)=1, \lim _{t \rightarrow 0^{+}} p(t) y^{\prime}(t)=0
\end{array}\right.
$$

and $u_{2}$ the unique solution to

$$
\left\{\begin{array}{l}
\frac{1}{p}\left(p y^{\prime}\right)^{\prime}+r(t) y=0 \text { a.e. on }[0,1] \\
y(0)=0, \lim _{t \rightarrow 0^{+}} p(t) y^{\prime}(t)=1 .
\end{array}\right.
$$


Now $u_{1}$ and $u_{2}$ are linearly independent and their Wronskian $W(t)$, at $t$, satisfies $p(t) W^{\prime}(t)+p^{\prime}(t) W(t)=0$ so $p(t) W(t)=$ constant $\neq 0, t \in[0,1]$. The general solution (method of variation of parameters) of

$$
\frac{1}{p}\left(p y^{\prime}\right)^{\prime}+r(t) y=g(t) \text { a.e. on }[0,1]
$$

is

$$
y(t)=d_{0} u_{1}(t)+d_{1} u_{2}(t)+\int_{0}^{t} \frac{\left[u_{2}(t) u_{1}(s)-u_{1}(t) u_{2}(s)\right]}{W(s)} g(s) d s
$$

where $d_{0}$ and $d_{1}$ are constants. The standard construction of the Green's function, see [17-18] for example, yields

Theorem 1.2: Let $B$ denote either $(S L),(N),(P)$ or $(B r)$ and $B_{0}$ either $(S L)_{0},(N)_{0},(P)$ or $(B r)_{0}$. Suppose (1.3) and (1.4) are satisfies. If

$$
\left\{\begin{array}{l}
\frac{1}{p}\left(p y^{\prime}\right)^{\prime}+r(t) y=0 \text { a.e. on }[0,1] \\
y \in B_{0}
\end{array}\right.
$$

has only the trivial solution, then

$$
\left\{\begin{array}{l}
\frac{1}{p}\left(p y^{\prime}\right)^{\prime}+r(t) y=0 \text { a.e. on }[0,1] \\
y \in B
\end{array}\right.
$$

has exactly one solution $y$, given by (1.6), where $d_{0}$ and $d_{1}$ are uniquely determined from the boundary condition. In fact,

$$
y(t)=A_{0} y_{1}(t)+A_{1} y_{2}(t)+\int_{0}^{1} G(t, s) g(s) d s
$$

where $G(t, s)$ is the Green's function and $A_{0}$ and $A_{1}$ are uniquely determined by the boundary conditions. Of course,

where $y_{1}$ and $y_{2}$ are the two "usual" linearly independent solutions i.e., choose $y_{1} \neq 0, y_{2} \neq 0$ so that $y_{1}, y_{2}$ satisfy $\frac{1}{p}\left(p y^{\prime}\right)^{\prime}+r(t) y=0$ a.e. on $[0,1]$ with $y_{1}$ satisfying the first boundary condition of $B_{0}$ and $y_{2}$ satisfying the second boundary condition of $B_{0}$.

Of course, analogue versions of theorems 1.1 and 1.2 hold for the more general problem

$$
\left\{\begin{array}{l}
\frac{1}{p}\left(p y^{\prime}\right)^{\prime}+r(t) y+\kappa(t) p(t) y^{\prime}(t)=g(t) \text { a.e. on }[0,1] \\
y \in(S L),(N),(P) \text { or }(B r)
\end{array}\right.
$$

where $\kappa$ satisfies 


$$
\kappa \in L_{p}^{1}[0,1]
$$

Theorem 1.3: If (1.3), (1.4) and (1.10) are satisfied and if

$$
\left\{\begin{array}{l}
\frac{1}{p}\left(p y^{\prime}\right)^{\prime}+r(t) y+\kappa(t) p(t) y^{\prime}(t)=0 \text { a.e. on }[0,1] \\
y \in(S L)_{0},(N)_{0},(P) \text { or }(B r)_{0}
\end{array}\right.
$$

has only the trivial solution, then (1.9) has exactly one solution given by (1.8) (where $G(t, s)$ is the appropriate Green's function).

In practice, one usually examines (1.7) and not the more general problem (1.9). This is due to the fact that numerical schemes [3] are available for Sturm Liouville eigenvalue problems (see section 4). However from a theoretical point of view, it is of interest to establish the most general result.

\section{Existence Principles}

We use a fixed point approach to establish our existence principles. In particular, we use a nonlinear alternative of Leray-Schauder type [9] which is an immediate consequence of the topological transversality theorem [8] of Granas. For completeness, we state the result. By a map being compact we mean it is continuous with relatively compact range. A map is completely continuous if it is continuous and the image of every bounded set in the domain is contained in a compact set of the range.

Theorem 2.1: (Nonlinear Alternative) Assume $U$ is a relatively open subset of a convex set $K$ in a Banach space $E$. Let $N: \bar{U} \rightarrow K$ be a compact map with $p \in U$. Then either

(i) $\quad N$ has a fixed point in $\bar{U}$; or

(ii) there is a point $u \in \partial U$ and $\lambda \in(0,1)$ such that $u=\lambda N u+(1-\lambda) p$.

Consider first the boundary value problem

$$
\left\{\begin{array}{l}
\frac{1}{p}\left(p y^{\prime}\right)^{\prime}+\tau(t) y=f\left(t, y, p y^{\prime}\right) \text { a.e. on }[0,1] \\
y \in(S L) \text { or }(N)
\end{array}\right.
$$

By a solution to $(2.1)$ we mean a function $y \in C[0,1] \cap C^{1}(0,1), p y^{\prime} \in A C[0,1]$ which satisfies the differential equation in $(2.1)$ a.e. on $[0,1]$ and the stated boundary conditions.

Theorem 2.2: Let $p f:[0,1] \times \mathbf{R}^{2} \rightarrow \mathbf{R}$ be an $L^{1}$-Carathéodory function and assume $p$ satisfies (1.3) and $\tau$ satisfies

In addition, suppose

$$
\tau \in L_{p}^{1}[0,1]
$$

$$
\left\{\begin{array}{l}
\frac{1}{p}\left(p y^{\prime}\right)^{\prime}+\tau y=0 \text { a.e. on }[0,1] \\
y \in(S L)_{0} \text { or }(N)_{0}
\end{array}\right.
$$

has only the trivial solution. Now suppose there is a constant $M_{0}$, independent of $\lambda$, with

$$
\|y\|_{1}=\max \left\{\sup _{[0,1]}|y(t)|, \sup _{(0,1)}\left|p(t) y^{\prime}(t)\right|\right\} \leq M_{0}
$$


for any solution $y$ to

$$
\left\{\begin{array}{l}
\frac{1}{p}\left(p y^{\prime}\right)^{\prime}+\tau(t) y=\lambda f\left(t, y, p y^{\prime}\right) \text { a.e. on }[0,1] \\
y \in(S L) \text { or }(N)
\end{array}\right.
$$

for each $\lambda \in(0,1)$. Then (2.1) has at least one solution.

Proof: Let $y_{1}$ and $y_{2}$ be two linearly independent solutions (see section 1) of $\left(p y^{\prime}\right)^{\prime}+\tau p y=0$ a.e. on $[0,1]$ with $y_{1}, y_{2} \in C[0,1]$ and $p y_{1}^{\prime}, p y_{2}^{\prime} \in A C[0,1]$.

Remark: In the analysis that follows, $(N)$ will be thought of as $(S L)$ with $\alpha=a=0$, $\beta=b=1$.

Choose $y_{2}$ so that $-\alpha y_{2}(0)+\beta \lim _{t \rightarrow 0^{+}} p(t) y_{2}^{\prime}(t) \neq 0$. If this is not possible, then the two linearly independent solutions are such that $-\alpha y_{1}(0)+\beta \lim _{t \rightarrow 0^{+}} p(t) y_{1}^{\prime}(t)=-\alpha y_{2}(0)+\beta \lim _{t \rightarrow 0^{+}}$
$p(t) y_{2}^{\prime}(t)=0$. Let

$$
u(x)=\left[a y_{2}(1)+b \lim _{t \rightarrow 1_{-}^{-}} p(t) y_{2}^{\prime}(t)\right] y_{1}(x)-\left[a y_{1}(1)+b \lim _{t \rightarrow 1_{-}} p(t) y_{1}^{\prime}(t)\right] y_{2}(x)
$$

so $u$ satisfies $\left(p u^{\prime}\right)^{\prime}+\tau p u=0$ a.e. on $[0,1]$ with $\quad-\alpha u(0)+\beta \lim _{t \rightarrow 0^{+}} p(t) u^{\prime}(t)=0$ and $a u(1)+b \lim _{t \rightarrow 1_{-}} p(t) u^{\prime}(t)=0$. Consequently, $u \equiv 0$, a contradiction since $y_{1}$ and $y_{2}$ are linearly independent. Solving $(2.4)_{\lambda}$ is equivalent to finding a $y \in C[0,1]$ with $p y^{\prime} \in C[0,1]$ which satisfies

$$
y(t)=A_{\lambda} y_{1}(t)+B_{\lambda} y_{2}(t)+\lambda \int_{0}^{t} \frac{\left[y_{1}(s) y_{2}(t)-y_{1}(t) y_{2}(s)\right]}{W(s)} f\left(s, y(s), p y^{\prime}\right) d s
$$

where $W(s)$ is the Wronskian of $y_{1}$ and $y_{2}$ at $s$ and

$$
B_{\lambda}=\frac{c_{0}-A_{\lambda} Q_{3}}{Q_{1}} \text { and } A_{\lambda}=\frac{c_{0} Q_{2}-c_{1} Q_{1}+\lambda Q_{5}}{Q_{3} Q_{2}-Q_{4} Q_{1}} \text {. }
$$

Here $\quad Q_{1}=-\alpha y_{2}(0)+\beta \operatorname{sim}_{t \rightarrow 0^{+}} p(t) y_{2}^{\prime}(t), \quad Q_{2}=a y_{2}(1)+b_{t \rightarrow 1_{-}} p(t) y_{2}^{\prime}(t), Q_{3}=-\alpha y_{1}(0)+$ $\beta \lim _{t \rightarrow 0^{+}} p(t) y_{1}^{\prime}(t)$ and $Q_{4}=a \underset{1}{a}(1)+\underset{t \rightarrow 0^{+}}{y_{1}} p(t) y_{1}^{\prime}(t)$ with

$$
\begin{gathered}
Q_{5}=a Q_{1} \int_{0}^{1} \frac{\left[y_{1}(s) y_{2}(1)-y_{1}(1) y_{2}(s)\right]}{W(s)} f\left(s, y(s), p(s) y^{\prime}(s)\right) d s \\
+b Q_{1} \int_{0}^{1} \frac{\left[y_{1}(s) \lim _{t \rightarrow 1} p(t) y_{2}^{\prime}(t)-y_{2}(s) \lim _{t \rightarrow 1} p(t) y_{1}^{\prime}(t)\right]}{W(s)} f\left(s, y(s), p(s) y^{\prime}(s)\right) d s .
\end{gathered}
$$

Remarks: $(\underline{i})$ Note $Q_{3} Q_{2}-Q_{4} Q_{1} \neq 0$. To see this, let $u(x)=Q_{1} y_{1}(x)-Q_{3} y_{2}(x)$. Notice $\left(p u^{\prime}\right)^{\prime}+\tau p u=0$ a.e. on $[0,1]$ and $-\alpha u(0)+\beta l i m, p(t) u^{\prime}(t)=0 . \quad$ If $Q_{3} Q_{2}-Q_{4} Q_{1}=0$ then $a u(1)+b_{t \rightarrow 1_{-}} p(t) u^{\prime}(t)=0$. Consequently, $u \equiv \underset{t \rightarrow 0}{0}$ i.e., $y_{1}(x)=\frac{Q_{3}}{Q_{1}} y_{2}(x)$, a contradiction. $\quad(\underline{i i})$ Since $p W^{\prime}+p^{\prime} W=0$ then $p W=$ constant.

We can rewrite (2.5) as 


$$
\begin{gathered}
y(t)=\lambda\left(C y_{1}(t)+D y_{2}(t)+\int_{0}^{t} \frac{\left[y_{1}(s) y_{2}(t)-y_{1}(t) y_{2}(s)\right]}{W(s)} f\left(s, y(s), p y^{\prime}\right) d s\right) \\
+(1-\lambda)\left[E y_{1}(t)+F y_{2}(t)\right]
\end{gathered}
$$

where

$$
F=\frac{c_{0}-E Q_{3}}{Q_{1}}, E=\frac{c_{0} Q_{2}-c_{1} Q_{1}}{Q_{3} Q_{2}-Q_{4} Q_{1}}, C=\frac{Q_{5}+c_{0} Q_{2}-c_{1} Q_{1}}{Q_{3} Q_{2}-Q_{4} Q_{1}} \text { and } D=\frac{c_{0}-C Q_{3}}{Q_{1}}
$$

Define the operator $N: K_{\mathscr{B}}^{1} \rightarrow K_{\mathscr{B}}^{1}$ by setting

$$
N y(t)=C y_{1}(t)+D y_{2}(t)+\int_{0}^{t} \frac{\left[y_{1}(s) y_{2}(t)-y_{1}(t) y_{2}(s)\right]}{W(s)} f\left(s, y(s), p y^{\prime}\right) d s .
$$

Here $K_{\mathscr{B}}^{1}=\left\{u \in C[0,1], p u^{\prime} \in C[0,1]: u \in(S L)\right.$ or $\left.(N)\right\}$. Then $(2.4)_{\lambda}$ is equivalent to the fixed problem

$$
y=\lambda N y+(1-\lambda) p
$$

where $p=E y_{1}(t)+F y_{2}(t)$. We claim that $N: K_{\mathscr{B}^{\prime}}^{1} \rightarrow K_{\mathscr{B}}^{1}$ is continuous and completely continuous. Let $u_{n} \rightarrow u$ in $K_{\mathscr{\sigma}}^{1}$, i.e., $u_{n} \rightarrow u$ and $p u_{n}^{\prime} \rightarrow p u^{\prime}$ uniformly on [0,1]. Thus there exists $r>0$ with $\left|u_{n}(t)\right| \leq r,\left|p(t) u_{n}^{\prime}(t)\right| \leq r,|u(t)| \leq r,\left|p(t) u^{\prime}(t)\right| \leq r$ for $t \in[0,1]$. By the above uniform convergence we have $p(t) f\left(t, u_{n}(t), p(t) u_{n}^{\prime}(t)\right) \rightarrow p(t) f\left(t, u(t), p(t) u^{\prime}(t)\right)$ pointwise a.e. on $[0,1]$. Also there exists an integrable function $h_{r}$ with

$$
\left|p(t) f\left(t, u_{n}(t), p(t) u_{n}^{\prime}(t)\right)\right| \leq h_{r}(t) \text { a.e. } t \in[0,1]
$$

Now

together with

$$
N u_{n}(t)=C y_{1}(t)+D y_{2}(t)+\int_{0}^{t} \frac{\left[y_{1}(s) y_{2}(t)-y_{1}(t) y_{2}(s)\right]}{W(s)} f\left(s, u_{n}(s), p u_{n}^{\prime} d s\right.
$$

$$
\begin{gathered}
p(t)\left(N u_{n}\right)^{\prime}(t)=C p(t) y_{1}^{\prime}(t) \\
+D p(t) y_{2}^{\prime}(t)+\int_{0}^{t} \frac{\left[y_{1}(s) p(t) y_{2}^{\prime}(t)-p(t) y_{1}^{\prime}(t) y_{2}(s)\right]}{W(s)} f\left(s, u_{n}(s), p u_{n}^{\prime}\right) d s
\end{gathered}
$$

and the Lebesgue dominated convergence theorem implies that $N u_{n} \rightarrow N u$ and $p\left(N u_{n}\right)^{\prime} \rightarrow p(N u)^{\prime}$ pointwise for each $t \in[0,1]$. In fact, the convergence is uniform because of (2.7). Consequently, $N u_{n} \rightarrow N u$ in $K_{\mathscr{B}}^{1}$ so $N$ is continuous. To see that $N$ is completely continuous, we use the ArzelaAscoli theorem. To see this, let $\Omega \subseteq K_{\mathscr{B}}^{1}$ be bounded, i.e., there exists a constant $M>0$ with $\|y\|_{1} \leq M$ for each $y \in \Omega$. Also there exist constants $C^{*}$ and $D^{*}$ (which may depend on $M$ ) such that $|C| \leq C^{*}$ and $|D| \leq D^{*}$ for all $y \in \Omega$. The boundedness of $N \Omega$ is immediate and to see the equicontinuity on $[0,1]$ consider $y \in \Omega$ and $t, z \in[0,1]$. Then

$$
\begin{gathered}
|N y(t)-N y(z)| \leq C^{*}\left|y_{1}(t)-y_{1}(z)\right|+D^{*}\left|y_{2}(t)-y_{2}(z)\right| \\
+\left|y_{2}(t)\right|\left|\int_{t}^{z} \frac{y_{1}(s)}{W(s)} f\left(s, y(s), p y^{\prime}\right) d s\right|
\end{gathered}
$$


and

$$
\begin{gathered}
+\left|y_{2}(t)-y_{2}(z)\right|\left|\int_{0}^{z} \frac{y_{1}(s)}{W(s)} f\left(s, y(s), p y^{\prime}\right) d s\right| \\
+\left|y_{1}(t)\right|\left|\int_{t}^{z} \frac{y_{2}(s)}{W(s)} f\left(s, y(s), p y^{\prime}\right) d s\right| \\
+\left|y_{1}(t)-y_{1}(z)\right|\left|\int_{0}^{z} \frac{y_{2}(s)}{W(s)} f\left(s, y(s), p y^{\prime}\right) d s\right|
\end{gathered}
$$

$$
\begin{gathered}
\left|p(t)(N y)^{\prime}(t)-p(z)(N y)^{\prime}(z)\right| \leq C^{*}\left|p(t) y_{1}^{\prime}(t)-p(z) y_{1}^{\prime}(z)\right| \\
+D^{*}\left|p(t) y_{2}^{\prime}(t)-p(z) y_{2}^{\prime}(z)\right| \\
+\left|p(t) y_{2}^{\prime}(t)\right|\left|\int_{t}^{z} \frac{y_{1}(s)}{W(s)} f\left(s, y(s), p y^{\prime}\right) d s\right| \\
+\left|p(t) y_{2}^{\prime}(t)-p(z) y_{2}^{\prime}(z)\right|\left|\int_{0}^{z} \frac{y_{1}(s)}{W(s)} f\left(s, y, p y^{\prime}\right) d s\right| \\
+\left|p(t) y_{1}^{\prime}(t)\right|\left|\int_{t}^{z} \frac{y_{2}(s)}{W(s)} f\left(s, y(s), p y^{\prime}\right) d s\right| \\
+\left|p(t) y_{1}^{\prime}(t)-p(z) y_{1}^{\prime}(z)\right|\left|\int_{0}^{z} \frac{y_{2}(s)}{W(s)} f\left(s, y, p y^{\prime}\right) d s\right|
\end{gathered}
$$

so the equicontinuity of $N \Omega$ follows from the above inequalities. Thus $N: K_{\mathscr{B}}^{1} \rightarrow K_{\mathscr{B}}^{1}$ is completely continuous. Set

$$
U=\left\{u \in K_{\mathscr{B}}^{1}:\|u\|_{1}<M_{0}+1\right\}, K=K_{\mathscr{B}}^{1} \text { and } E=\left\{u \in C[0,1] \text { with } p u^{\prime} \in C[0,1]\right\}
$$

Then theorem 2.1 implies that $N$ has a fixed point, i.e. (2.1) has a solution $y \in C[0,1]$ with $p y^{\prime} \in C[0,1]$. The fact that $p y^{\prime} \in A C[0,1]$ follows from (2.5) with $\lambda=1$.

We next consider the problem

$$
\left\{\begin{array}{l}
\frac{1}{p}\left(p y^{\prime}\right)^{\prime}+\tau(t) y=f\left(t, y, p y^{\prime}\right) \text { a.e. on }[0,1] \\
y \in(P) .
\end{array}\right.
$$

Theorem 2.3: Let $p f:[0,1] \times \mathbf{R}^{2} \rightarrow \mathbf{R}$ be an $L^{1}$-Carathéodory function and assume (1.3) and (2.2) hold. In addition, suppose

$$
\left\{\begin{array}{l}
\frac{1}{p}\left(p y^{\prime}\right)^{\prime}+\tau y=0 \text { a.e. on }[0,1] \\
y \in(P)
\end{array}\right.
$$


has only the trivial solution. Also suppose there is a constant $M_{0}$, independent of $\lambda$, with $\|y\|_{1} \leq M_{0}$ for any solution $y$ to

$$
\left\{\begin{array}{l}
\frac{1}{p}\left(p y^{\prime}\right)^{\prime}+\tau(t) y=\lambda f\left(t, y, p y^{\prime}\right) \text { a.e. on }[0,1] \\
y \in(P)
\end{array}\right.
$$

for each $\lambda \in(0,1)$. Then $(2.8)$ has at least one solution.

Proof: Let $y_{1}$ and $y_{2}$ be two linearly independent solutions of $\left(p y^{\prime}\right)^{\prime}+\tau p y=0$ with $y_{1}, y_{2} \in C[0,1]$ and $p y_{1}^{\prime}, p y_{2}^{\prime} \in A C[0,1]$. Choose $y_{2}$ with $y_{2}(0)-y_{2}(1) \neq 0$.

If this is not possible, then the two linearly independent solutions are such that $y_{2}(0)-y_{2}(1)=y_{1}(0)-y_{1}(1)=0$. Let

$$
\begin{gathered}
u(x)=\left[\lim _{t \rightarrow 0^{+}} p(t) y_{2}^{\prime}(t)-\lim _{t \rightarrow 1_{-}^{-}} p(t) y_{2}^{\prime}(t)\right] y_{1}(x) \\
-\left[\lim _{t \rightarrow 0^{+}} p(t) y_{1}^{\prime}(t)-\lim _{t \rightarrow 1_{-}^{-}} p(t) y_{1}^{\prime}(t)\right] y_{2}(x)
\end{gathered}
$$

so $u$ satisfies $\left(p u^{\prime}\right)^{\prime}+\tau p u=0$ a.e. on $[0,1]$ with $u(0)=u(1)$ and $\lim _{t \rightarrow 0^{+}} p(t) u^{\prime}(t)=\lim _{t \rightarrow 1_{-}} p(t) u^{\prime}(t)$. Consequently, $u \equiv 0$, a contradiction since $y_{1}$ and $y_{2}$ are linearly independent. where

Solving $(2.9)_{\lambda}$ is equivalent to finding a $y \in C[0,1]$ with $p y^{\prime} \in C[0,1]$ which satisfies (2.5)

$$
B_{\lambda}=\frac{A_{\lambda}\left[y_{1}(1)-y_{1}(0)\right]+\lambda I_{2}}{y_{2}(0)-y_{2}(1)}
$$

and

$$
A_{\lambda}=\frac{\lambda\left[I_{2}+I_{3}\right]}{\left[y_{2}(0)-y_{2}(1)\right] I_{0}-\left[y_{1}(1)-y_{1}(0)\right] I_{1}}
$$

Here $I_{0}=\lim _{t \rightarrow 0^{+}} p(t) y_{1}^{\prime}(t)-\lim _{t \rightarrow 1_{-}} p(t) y_{1}^{\prime}(t), I_{1}=\lim _{t \rightarrow 0^{+}} p(t) y_{2}^{\prime}(t)-\lim _{t \rightarrow 1_{-}} p(t) y_{2}^{\prime}(t)$ with

and

$$
I_{2}=\int_{0}^{1} \frac{\left[y_{1}(s) y_{2}(1)-y_{1}(1) y_{2}(s)\right]}{W(s)} f\left(s, y(s), p(s) y^{\prime}(s)\right) d s
$$

$I_{3}=\left[y_{2}(0)-y_{2}(1)\right] \int_{0}^{1} \frac{\left[y_{1}(s) \lim _{t \rightarrow 1} p(t) y_{2}^{\prime}(t)-y_{2}(s) \lim _{t \rightarrow 1} p(t) y_{1}^{\prime}(t)\right]}{W(s)} f\left(s, y(s), p y^{\prime}\right) d s$.

Remark: Notice $\left[y_{2}(0)-y_{2}(1)\right] I_{0}-\left[y_{1}(1)-y_{1}(0)\right] I_{1} \neq 0$ for if not, then

$$
u(x)=y_{1}(x)+\frac{\left[y_{1}(1)-y_{1}(0)\right]}{\left[y_{2}(0)-y_{2}(1)\right]} y_{2}(x)
$$

satisfies $\left(p u^{\prime}\right)^{\prime}+\tau p u=0$ a.e. on $[0,1]$ with $u(0)=u(1)$ and $\lim _{t \rightarrow 0^{+}} p(t) u^{\prime}(t)=\lim _{t \rightarrow 1} p(t) u^{\prime}(t)$.
Then $u \equiv 0$, a contradiction.

Essentially, the same reasoning as in theorem 2.2 establishes the result.

Next consider the problem 


$$
\left\{\begin{array}{l}
\frac{1}{p}\left(p y^{\prime}\right)^{\prime}+\tau(t) y=f\left(t, y, p y^{\prime}\right) \text { a.e. on }[0,1] \\
y \in(B r)
\end{array}\right.
$$

Theorem 2.4: Let $p f:[0,1] \times \mathbf{R}^{2} \rightarrow \mathbf{R}$ be a Carathéodory function and assume (1.3) and (2.2) hold. In addition, suppose

$$
\left\{\begin{array}{l}
\frac{1}{p}\left(p y^{\prime}\right)^{\prime}+\tau y=0 \text { a.e. on }[0,1] \\
y \in(B r)_{0}
\end{array}\right.
$$

has only the trivial solution. Also, suppose there is a constant $M_{0}$, independent of $\lambda$, with $\|y\|_{1} \leq M_{0}$ for any solution $y$ to

$$
\left\{\begin{array}{l}
\frac{1}{p}\left(p y^{\prime}\right)^{\prime}+\tau(t) y=\lambda f\left(t, y, p y^{\prime}\right) \text { a.e. on }[0,1] \\
y \in(B r)
\end{array}\right.
$$

for each $\lambda \in(0,1)$. Then $(2.10)$ has at least one solution.

Proof: Let $y_{1}$ and $y_{2}$ be two linearly independent solutions of $\left(p y^{\prime}\right)^{\prime}+\tau p y=0$ with $y_{1}, y_{2} \in C[0,1]$ and $p y_{1}^{\prime}, p y_{2}^{\prime} \in A C[0,1]$. Choose $y_{2}$ with $y_{2}(0) \neq 0$. Solving $(2.11)_{\lambda}$ is equivalent to finding a $y \in C[0,1]$ with $p y^{\prime} \in C[0,1]$ which satisfies $(2.5)$ where

$$
B_{\lambda}=\frac{c_{0}-A_{\lambda} y_{1}(0)}{y_{2}(0)}
$$

and

with

$$
C=\int_{0}^{1} \frac{d s}{p(s)} \lim _{t \rightarrow 1_{-}} p(t) y_{1}^{\prime}(t)-\left(\frac{y_{1}(0)}{y_{2}(0)}\right) \int_{0}^{1} \frac{d s}{p(s)_{t \rightarrow 1_{-}}} \lim _{-}(t) y_{2}^{\prime}(t)-y_{1}(1)+\left(\frac{y_{1}(0)}{y_{2}(0)}\right) y_{2}(1)
$$

$$
\begin{aligned}
& A_{\lambda}=\frac{1}{C}\left(c_{1}+\int_{0}^{1} \frac{d s}{p(s)}\left[\lambda \int_{0}^{1} \frac{\left[y_{1}(s) \lim _{t \rightarrow 1} p(t) y_{2}^{\prime}(t)-y_{2}(s) \lim _{t \rightarrow 1} p(t) y_{1}^{\prime}(t)\right]}{W(s)} f\left(s, y(s), p y^{\prime}\right) d s\right.\right. \\
& \left.\left.-\frac{c_{0_{t \rightarrow 1}} \lim _{-} p(t) y_{2}^{\prime}(t)}{y_{2}(0)}\right]+\frac{c_{0} y_{2}(0)}{y_{2}(0)}+\lambda \int_{0}^{1} \frac{\left[y_{1}(s) y_{2}(1)-y_{2}(s) y_{1}(1)\right]}{W(s)} f\left(s, y(s), p(s) y^{\prime}(s)\right) d s\right) .
\end{aligned}
$$

Remark: Notice $C \neq 0$. To see this, let

$$
u(x)=y_{1}(x)-\left(\frac{y_{1}(0)}{y_{2}(0)}\right) y_{2}(x)
$$

so $\quad C=\int_{0}^{1} \frac{d s}{p(s)} \lim _{t \rightarrow 1} p(t) u^{\prime}(t)-u(1) . \quad$ Now $\quad\left(p u^{\prime}\right)^{\prime}+\tau p u=0 \quad$ a.e. $\quad$ on $\quad[0,1] \quad$ with $\quad u(0)=0$. If $C=0$ then, $\int_{0}^{1} \frac{d s}{p(s)} \lim _{t \rightarrow 1} p(t) u^{\prime}(t)-u(1)=0$. Consequently, $u \equiv 0$, a contradiction.

Essentially the same reasoning as in theorem 2.2 establishes the result.

Of course, more general forms of theorems $2.2,2.3$ and 2.4 are immediately available for us for the boundary value problem

$$
\left\{\begin{array}{l}
\frac{1}{p}\left(p y^{\prime}\right)^{\prime}+\tau(t) y+\sigma(t) p y^{\prime}=f\left(t, y, p y^{\prime}\right) \text { a.e. on }[0,1] \\
y \in(S L) \text { or }(N) \text { or }(P) \text { or }(B r)
\end{array}\right.
$$


Theorem 2.5: Let $p f:[0,1] \times \mathbf{R} \rightarrow \mathbf{R}$ be an $L^{1}$-Carathéodory function and assume (1.3) and (2.2) hold with $\sigma$ satisfying

$$
\sigma \in L_{p}^{1}[0,1]
$$

In addition, suppose

$$
\left\{\begin{array}{l}
\frac{1}{p}\left(p y^{\prime}\right)^{\prime}+\tau y+\sigma p y^{\prime}=0 \text { a.e. on }[0,1] \\
y \in(S L)_{0} \text { or }(N)_{0} \text { or }(P) \text { or }(B r)_{0}
\end{array}\right.
$$

has only the trivial solution. Also suppose there is a constant $M_{0}$, independent of $\lambda$, with $\|y\|_{1} \leq M_{0}$ for any solution $y$ to

$$
\left\{\begin{array}{l}
\frac{1}{p}\left(p y^{\prime}\right)^{\prime}+\tau(t) y+\sigma(t) p y^{\prime}=\lambda f\left(t, y, p y^{\prime}\right) \text { a.e. on }[0,1] \\
y \in(S L) \text { or }(N) \text { or }(P) \text { or }(B r)
\end{array}\right.
$$

for each $\lambda \in(0,1)$. Then $(2.12)$ has at least one solution.

Proof: Essentially the same reasoning as in theorems 2.2, 2.3 and 2.4 establishes the result.

\section{Existence Theory}

We begin by establishing an existence result for the boundary value problem

$$
\left\{\begin{array}{l}
\frac{1}{p}\left(p y^{\prime}\right)^{\prime}+\tau(t) y=f\left(t, y, p y^{\prime}\right) \text { a.e. on }[0,1] \\
y \in(S L) \text { or }(N) \text { or }(P) \text { or }(B r) .
\end{array}\right.
$$

Theorem 3.1: Let $p f:[0,1] \times \mathbf{R}^{2} \rightarrow \mathbf{R}$ be an $L^{1}$-Carathéodory function and assume (1.3) and (2.2) hold. In addition, suppose

$$
\left\{\begin{array}{l}
\frac{1}{p}\left(p y^{\prime}\right)^{\prime}+\tau y=0 \text { a.e. on }[0,1] \\
y \in(S L)_{0} \text { or }(N)_{0} \text { or }(P) \text { or }(B r)_{0}
\end{array}\right.
$$

has only the trivial solution. Let $f(t, u, v)=n v+g(t, u, v)$ and assume

$$
n \in L_{p}^{1}[0,1]
$$

$$
\left\{\begin{array}{c}
p g \text { is an } L^{1} \text {-Carathéodory function and } \\
|g(t, u, v)| \leq \phi_{1}(t)+\phi_{2}(t)|u|^{\gamma}+\phi_{3}(t)|v|^{\theta} \\
\text { for a.e. } t \in[0,1], \text { for constants } \gamma, \theta \text { with } 0 \leq \gamma, \theta<1 \\
\text { and functions } \phi_{i} \in L_{p}^{1}[0,1], \quad i=1,2,3
\end{array}\right.
$$

and 


$$
\left\{\begin{array}{c}
\sup _{t \in[0,1]} \int_{0}^{1}\left|p(t) G_{t}(t, s) n(s)\right| d s<1 \\
\text { Here } G(t, s) \text { is the Green's function associated with } \\
\left(p y^{\prime}\right)+p r y=0 \text { a.e. on }[0,1] \text { with } y \in(S L) \text { or }(N) \text { or }(P) \text { or }(B r)
\end{array}\right.
$$

hold. Then (3.1) has at least one solution.

Remark: Since $p W^{\prime}+p^{\prime} W=0$ then $\sup _{t \in[0,1]}\left|p(t) G_{t}(t, s)\right| \leq E_{0} p(s)$ for some constant $E_{0}$.

Proof: Let $y$ be a solution to

$$
t \in[0,1]
$$

$$
\left\{\begin{array}{l}
\frac{1}{p}\left(p y^{\prime}\right)^{\prime}+\tau(t) y=\lambda f\left(t, y, p y^{\prime}\right) \text { a.e. on }[0,1] \\
y \in(S L) \text { or }(N) \text { or }(P) \text { or }(B r)
\end{array}\right.
$$

for $0<\lambda<1$. Then

$$
y(t)=y_{3}(t)+\int_{0}^{1} G(t, s) f\left(s, y(s), p(s) y^{\prime}(s)\right) d s, \quad t \in[0,1]
$$

where $y_{3}$ is the unique solution of $\left(p y^{\prime}\right)^{\prime}+p \tau y=0$ a.e. on $[0,1]$ with $y \in(S L)$ or $(N)$ or $(P)$ or $(B r)$ and $G(t, s)$ is as described in (3.5). Also notice

$$
p(t) y^{\prime}(t)=p(t) y_{3}^{\prime}(t)+\int_{0}^{1} p(t) G_{t}(t, s) f\left(s, y(s), p(s) y^{\prime}(s)\right) d s .
$$

Now (3.4) together with (3.7) yields

$$
\begin{aligned}
& |y|_{0} \equiv \sup _{[0,1]}|y(t)| \leq \sup _{[0,1]}\left|y_{3}(t)\right| \\
& +\left|p y^{\prime}\right|_{\substack{0 \\
t \in[0,1]}}^{t} \int_{0}^{t}|G(t, s) n(s)| d s+\sup _{t \in[0,1]} \int_{0}^{t}\left|G(t, s) \phi_{1}(s)\right| d s \\
& +|y| \underset{t \in[0,1]}{\gamma} \int_{0}^{t}\left|G(t, s) \phi_{2}(s)\right| d s+\left|p y^{\prime}\right|_{\substack{0 \\
t \in[0,1]}}^{\theta} \int_{0}^{t}\left|G(t, s) \phi_{3}(s)\right| d s
\end{aligned}
$$

so there exist constants $A_{0}, A_{1}, A_{2}$ and $A_{3}$ with

$$
|y|_{0} \leq A_{0}+A_{1}\left|p y^{\prime}\right|_{0}+A_{2}|y|_{0}^{\gamma}+A_{3}\left|p y^{\prime}\right|_{0}^{\theta} .
$$

Also there exists a constant $A_{4}>0$ with

$$
A_{2} x^{\gamma} \leq \frac{1}{2} x+A_{4} \text { for all } x>0 .
$$

Putting this into (3.9) yields

$$
|y|_{0} \leq 2\left(A_{0}+A_{4}\right)+2 A_{1}\left|p y^{\prime}\right|_{0}+2 A_{3}\left|p y^{\prime}\right|_{0}^{\theta} .
$$


Also (3.8) implies that there are constants $A_{5}, A_{6}$, and $A_{7}$. with

$$
\begin{gathered}
\left|p y^{\prime}\right|_{0} \leq A_{5}+\left|p y^{\prime}\right|_{0}\left(\sup _{t \in[0,1]} \int_{0}^{t}\left|p(t) G_{t}(t, s) n(s)\right| d s\right) \\
+A_{6}|y|_{0}^{\gamma}+A_{7}\left|p y^{\prime}\right|_{0}^{\theta} .
\end{gathered}
$$

Put (3.10) into (3.11) to obtain

$$
\begin{aligned}
& \left|p y^{\prime}\right|_{0} \leq A_{5}+\left|p y^{\prime}\right|_{0}\left(\sup _{t \in[0,1]} \int_{0}^{t}\left|p(t) G_{t}(t, s) n(s)\right| d s\right) \\
& +A_{7}\left|p y^{\prime}\right|_{0}^{\theta}+A_{6}\left(2\left(A_{0}+A_{4}\right)+2 A_{1}\left|p y^{\prime}\right|_{0}+2 A_{3}\left|p y^{\prime}\right| \begin{array}{l}
\theta \\
0
\end{array}\right)^{\gamma}
\end{aligned}
$$

and so there exist constants $A_{8}, A_{9}, A_{10}$, and $A_{11}$ with

$$
\begin{aligned}
& \left(\underset{t \in[0,1]}{1-\sup _{0}^{t}} \int_{t}^{t}\left|p(t) G_{t}(t, s) n(s)\right| d s\right)\left|p y^{\prime}\right|_{0} \\
& \leq A_{8}+A_{9}\left|p y^{\prime}\right|_{0}^{\gamma}+A_{10}\left|p y^{\prime}\right|_{0}^{\gamma \theta}+A_{11}\left|p y^{\prime}\right|_{0}^{\theta} .
\end{aligned}
$$

Consequently there exists a constant $M_{0}^{*}$, independent of $\lambda$, with $\left|p y^{\prime}\right|_{0} \leq M_{0}^{*}$. This together with (3.10) yields the existence of a constant $M_{0}^{* *}$ with $|y|_{0} \leq M_{0}^{* *}$. Let $M_{0}=\max \left\{M_{0}^{*}, M_{0}^{* *}\right\}$ and this together with either theorems $2.2,2.3$ or 2.4 establishes the result.

Consider the Sturm Liouville eigenvalue problem

$$
\left\{\begin{array}{l}
L u=\lambda u \text { a.e. on }[0,1] \\
u \in(S L)_{0} \text { or }(N)_{0} \text { or }(P) \text { or }(B r)_{0}
\end{array}\right.
$$

where $L u=-\frac{1}{p q(t)}\left[\left(p u^{\prime}\right)^{\prime}+r(t) p u\right]$, with $p$ satisfying (1.3) and

$$
r, q \in L_{p}^{1}[0,1] \text { with } q>0 \text { a.e. on }[0,1] .
$$

Then $L$ has a countably infinite number of real eigenvalues (see section 4 ) and it is possible to estimate these eigenvalues numerically [3].

Theorem 3.1 immediately yields an existence result for

$$
\left\{\begin{array}{l}
\frac{1}{p}\left(p y^{\prime}\right)^{\prime}+r(t) y+\mu q(t) y=f\left(t, y, p y^{\prime}\right) \text { a.e. on }[0,1] \\
y \in(S L) \text { or }(N) \text { or }(P) \text { or }(B r)
\end{array}\right.
$$

where $\mu$ is not an eigenvalue of (3.12).

Theorem 3.2: Let $p f:[0,1] \times \mathbf{R}^{2} \rightarrow \mathbf{R}$ be an $L^{1}$-Carathéodory function and assume (1.3) and (3.13) hold. Let $f(t, u, v)=n v+g(t, u, v)$ and assume (3.3), (3.4) and (3.5), with $\tau(t)=r(t)+$ $\mu q(t)$, are satisfied. Then (3.14) has at least one solution. 
Proof: Let $\tau(t)=r(t)+\mu q(t)$ in theorem 3.1.

Next in this section we obtain an existence result for the boundary value problem

$$
\left\{\begin{array}{l}
\frac{1}{p}\left(p y^{\prime}\right)^{\prime}+\tau(t) y+\sigma(t) p y^{\prime}=g\left(t, y, p y^{\prime}\right) \text { a.e. on }[0,1] \\
y \in(S L) \text { or }(N) \text { or }(P) \text { or }(B r) .
\end{array}\right.
$$

Theorem 3.3: Let pg: $[0,1] \times \mathbf{R}^{2} \rightarrow \mathbf{R}$ be an $L^{1}$-Carathéordory function and assume (1.3), (2.2), (2.13) and (3.4) hold. In addition, suppose

$$
\left\{\begin{array}{l}
\frac{1}{p}\left(p y^{\prime}\right)^{\prime}+\tau y+\sigma p y^{\prime}=0 \text { a.e. on }[0,1] \\
y \in(S L)_{0} \text { or }(N)_{0} \text { or }(P) \text { or }(B r)_{0}
\end{array}\right.
$$

has only the trivial solution. Then (3.15) has at least one solution.

Proof: Essentially the same argument as in theorem 3.1 (except easier) yields the result.

Remark: We remark here that theorem 3.2 seems to be the most applicable result in this paper since it is possible to estimate numerically [3] the eigenvalues of (3.12).

Finally we obtain a more subtle existence result for

$$
\left\{\begin{array}{l}
\frac{1}{p}\left(p y^{\prime}\right)^{\prime}+\tau y=g\left(t, y, p y^{\prime}\right) y+h\left(t, y, p y^{\prime}\right) \equiv f\left(t, y, p y^{\prime}\right) \text { a.e. on }[0,1] \\
y(0)=y(1)=0 .
\end{array}\right.
$$

Theorem 3.4: Let $p g, p h:[0,1] \times \boldsymbol{R}^{2} \rightarrow \boldsymbol{R}$ be $L^{1}$-Carathéodory functions and assume (1.3) and (2.2) hold. In addition, suppose (2.3), with $\beta=b=0$, has only the trivial solution. Also assume

$$
\left\{\begin{array}{c}
|h(t, u, v)| \leq \phi_{1}(t)+\phi_{2}(t)|u|^{\gamma}+\phi_{3}(t)|v|^{\theta} \\
\text { for a.e. } t \in[0,1] \text { with } 0 \leq \gamma, \theta<1
\end{array}\right.
$$

$$
\begin{gathered}
\text { there exist } \tau_{1}, \tau_{2} \in L_{p}^{1}[0,1] \text { with } \tau_{1}(t) \leq g(t, u, v) \leq \tau_{2}(t) \text { for a.e. } \\
t \in[0,1] ; \text { here } \tau_{1} \leq 0 \text { for a.e. } t \in[0,1] \text { and } \tau_{2} \geq 0 \text { for a.e. } t \in[0,1] \\
\phi_{1}, \phi_{2} \in L_{p}^{1}[0,1] \text { with } \int_{0}^{1}[p(t)]^{\frac{2+\theta}{2-\theta}}\left[\phi_{3}(t)\right]^{\frac{2}{2-\theta}} d t<\infty
\end{gathered}
$$

and

$$
\left\{\begin{array}{c}
W_{p}^{1,2}[0,1]=\Omega \oplus \Gamma \text { where } \Omega \subseteq K^{*} \text { is finite dimensional and for every } \\
0 \neq y=u+v \in K^{*} \text { with } u \in \Omega, v \in \Gamma \text { we have } R(y)>0 ; \Gamma=\Omega \perp
\end{array}\right.
$$

hold; here

$$
R(y)=\int_{0}^{1}\left[p\left(v^{\prime}\right)^{2}-\left(\tau-\tau_{1}\right) p v^{2}\right] d t-\int_{0}^{1}\left[p\left(u^{\prime}\right)^{2}-\left(\tau-\tau_{2}\right) p u^{2}\right] d t
$$


and

$$
K^{*}=\left\{w:[0,1] \rightarrow \boldsymbol{R}: w \in A C[0,1] \text { with } w^{\prime} \in L_{p}^{2}[0,1] \text { and } w(0)=w(1)\right\} .
$$

Then (3.16) has at least one solution.

Remark: (i) In (3.20) we have $y=u+v$ with $u \in \Omega, v \in \Gamma$ so $\int_{0}^{1} p u v d t+\int_{0}^{1} p u^{\prime} v^{\prime} d t=0$.

(ii) For notational purposes, let $\|u\|_{p}=\left(\int_{0}^{1} p|u|^{2} d t\right)^{\frac{1}{2}}$.

(iii) Recall by $W_{p}^{1,2}[0,1]$ we mean the space of functions $u \in A C[0,1]$ with $u^{\prime} \in L_{p}^{2}[0,1]$ and with norm

$$
\|u\|_{*}=\left(\int_{0}^{1} p|u|^{2} d t+\int_{0}^{1} p\left|u^{\prime}\right|^{2} d t\right)^{\frac{1}{2}} .
$$

Proof: First recall Lemma 2.8 in [7] implies there exists $\epsilon>0$ with

$$
R(y) \geq \epsilon\left(\|y\|_{p}^{2}+\left\|y^{\prime}\right\|_{p}^{2}\right)
$$

for any $y \in K^{*}$; here $y=u+v$ with $u \in \Omega$ and $v \in \Gamma$. Let $y(=u+v)$ be a solution to

$$
\left\{\begin{aligned}
\frac{1}{p}\left(p y^{\prime}\right)^{\prime}+\tau y & =\lambda f\left(t, y, p y^{\prime}\right) \text { a.e. on }[0,1] \\
y(0)=y(1) & =0
\end{aligned}\right.
$$

for some $0<\lambda<1$. Then

$$
\begin{gathered}
-\int_{0}^{1}(v-u)\left[\left(p y^{\prime}\right)^{\prime}+p \tau y\right] d t=-\lambda \int_{0}^{1} p(v-u) y g\left(t, y, p y^{\prime}\right) d t \\
-\lambda \int_{0}^{1} p(v-u) h\left(t, y, p y^{\prime}\right) d t
\end{gathered}
$$

and so integration by parts yields

$$
\begin{gathered}
\int_{0}^{1}\left[p\left(v^{\prime}\right)^{2}+p v^{2}\left(-\tau+\lambda g\left(t, y, p y^{\prime}\right)\right)\right] d t \\
-\int_{0}^{1}\left[p\left(u^{\prime}\right)^{2}+p u^{2}\left(-\tau+\lambda g\left(t, y, p y^{\prime}\right)\right)\right] d t \\
\leq \int_{0}^{1} p|v-u|\left|h\left(t, y, p y^{\prime}\right)\right| d t .
\end{gathered}
$$

Also

$$
p v^{2}\left[-\tau+\lambda g\left(t, y, p y^{\prime}\right)\right]=p v^{2}\left[-\left(\tau-\tau_{1}\right)+\lambda g\left(t, y, p y^{\prime}\right)-\tau_{1}\right]
$$




$$
\geq p v^{2}\left[-\left(\tau-\tau_{1}\right)+(\lambda-1) \tau_{1}\right] \geq-p\left(\tau-\tau_{1}\right) v^{2} \text { a.e. on }[0,1]
$$

Similarly

$$
p u^{2}\left[-\tau+\lambda g\left(t, y, p y^{\prime}\right)\right] \leq-p\left(\tau-\tau_{2}\right) u^{2} a . e . \text { on }[0,1]
$$

Putting this into (3.23) yields

$$
R(y) \leq \int_{0}^{1} p|v-u|\left|h\left(t, y, p y^{\prime}\right)\right| d t
$$

This together with (3.21) implies that there is an $\epsilon>0$ with

$$
\epsilon\left(\|y\|_{p}^{2}+\left\|y^{\prime}\right\|_{p}^{2}\right) \leq \int_{0}^{1} p|v-u|\left|h\left(t, y, p y^{\prime}\right)\right| d t .
$$

We also have $\|v-u\|_{p}^{2}+\left\|v^{\prime}-u^{\prime}\right\|_{p}^{2}=\|y\|_{p}^{2}+\left\|y^{\prime}\right\|_{p}^{2}$. Now Sobolev's inequality (since we have the imbedding $\left.W_{p}^{1,2}[0,1] \rightarrow C[0,1]\right)$ implies

$$
\int_{0}^{1} p \phi_{1}|v-u| d t \leq|v-u|_{0} \int_{0}^{1} p \phi_{1} d t \leq F_{1}\left(\|v-u\|_{p}^{2}+\left\|v^{\prime}-u^{\prime}\right\|_{p}^{2}\right)^{\frac{1}{2}}
$$

for some constant $F_{1}$. Thus

$$
\int_{0}^{1} p \phi_{1}|v-u| d t \leq F_{1}\left(\|y\|_{p}+\left\|y^{\prime}\right\|_{p}\right)
$$

Also

$$
\begin{aligned}
& \int_{0}^{1} p \phi_{2}|v-u||y|^{\gamma} d t \leq|v-u|_{0}|y|_{0}^{\gamma} \int_{0}^{1} p \phi_{2} d t \\
\leq & F_{2}\left(\|v-u\|_{p}^{2}+\left\|v^{\prime}-u^{\prime}\right\|_{p}^{2}\right)^{\frac{1}{2}}\left(\|y\|_{p}^{2}+\left\|y^{\prime}\right\|_{p}^{2}\right)^{\frac{\gamma}{2}}
\end{aligned}
$$

for some constant $F_{2}$. Thus there exists a constant $F_{3}$ with

$$
\int_{0}^{1} p \phi_{2}|v-u||y|^{\gamma} d t \leq F_{3}\left(\|y\|_{p}^{\gamma+1}+\left\|y^{\prime}\right\|_{p}^{\gamma+1}\right)
$$

Finally Hölder's inequality implies that there is a constant $F_{4}$ with

$$
\begin{gathered}
\int_{0}^{1} p \phi_{3}|v-u|\left|p y^{\prime}\right|^{\theta} d t \leq|v-u|_{0}\left\|y^{\prime}\right\|_{p}^{\theta}\left(\int_{0}^{1}[p(t)]^{\frac{2+\theta}{2-\theta}}\left[\phi_{3}(t)\right]^{\frac{2}{2-\theta}} d t\right)^{\frac{2-\theta}{2}} \\
\leq F_{4}\left\|y^{\prime}\right\|_{p}^{\theta}\left(\|v-u\|_{p}^{2}+\left\|v^{\prime}-u^{\prime}\right\|_{p}^{2}\right)^{\frac{1}{2}} .
\end{gathered}
$$

Thus there exists a constant $F_{5}$ with 


$$
\int_{0}^{1} p \phi_{3}|v-u|\left|p y^{\prime}\right|^{\theta} d t \leq F_{5}\left(\|y\|_{p}^{\theta+1}+\left\|y^{\prime}\right\|_{p}^{\theta+1}\right)
$$

Put (3.25), (3.26) and (3.27) into (3.24) and since $\theta, \gamma<1$ there exists a constant $F_{6}$ with

$$
\frac{\epsilon}{2}\left(\|y\|_{p}^{2}+\left\|y^{\prime}\right\|_{p}^{2}\right) \leq F_{6}
$$

Thus for $t \in[0,1]$,

$$
|y(t)| \leq \int_{0}^{1}\left|y^{\prime}(s)\right| d s \leq\left\|y^{\prime}\right\|_{p}\left(\int_{0}^{1} \frac{d s}{p(s)}\right)^{\frac{1}{2}}
$$

and this together with (3.28) implies that there exists a constant $F_{7}$, independent of $\lambda$, with

$$
|y|_{0}=\sup _{[0,1]}|y(t)| \leq F_{7},\|y\|_{p} \leq F_{7},\left\|y^{\prime}\right\|_{p} \leq F_{7}
$$

for any solution $y$ to $(3.22)_{\lambda}$.

This bound together with $(3.22)_{\lambda}$ implies there exist a constant $F_{8}$ with

$$
\int_{0}^{1}\left|\left(p y^{\prime}\right)^{\prime}\right| d t \leq F_{8}+\int_{0}^{1} p \phi_{3}\left|p y^{\prime}\right|^{\theta} d t .
$$

Hölder's inequality implies there exist a constant $F_{9}$ with

$$
\int_{0}^{1}\left|\left(p y^{\prime}\right)^{\prime}\right| d t \leq F_{8}+F_{9}\left\|y^{\prime}\right\|_{p}^{\theta} \leq F_{8}+F_{9} F_{7}^{\theta} \equiv F_{10} .
$$

Also there exist $t_{0} \in(0,1)$ with $y^{\prime}\left(t_{0}\right)=0$ so

Thus

$$
\left|p(t) y^{\prime}(t)\right| \leq\left|\int_{t_{0}}^{t}\right|\left(p y^{\prime}\right)^{\prime}|d t| \leq F_{10}
$$

$$
\sup _{(0,1)}\left|p(t) y^{\prime}(t)\right| \leq F_{10} .
$$

Theorem 2.2 together with (3.29) and (3.30) completes the proof.

\section{Appendix-Eigenvalues}

We now use the ideas of section 2 and results on compact self adjoint operators to give a unified treatment of the Sturm Liouville eigenvalue problem.

In particular, consider 


$$
\left\{\begin{array}{l}
L u=\lambda u \text { a.e. on }[0,1] \\
u \in(S L)_{0} \text { or }(N)_{0} \text { or }(P) \text { or }(B r)_{0}
\end{array}\right.
$$

where $L u=-\frac{1}{p q(t)}\left[\left(p u^{\prime}\right)^{\prime}+r(t) p u\right]$ and assume (1.3) and (3.13) hold. We first show that there exists $\lambda^{*} \in \mathbf{R}$ such that $\lambda^{*}$ is not an eigenvalue of (4.1).

Remark: If $r \equiv 0$ and $y \in(S L)_{0}$, then $\lambda^{*}=0$ will work whereas if $r \equiv 0$ and $y \in(N)_{0}$ or $(P)$ or $(B r)_{0}$, then $\lambda^{*}=-1$ will work.

Let

$$
D(L)=\left\{w \in C[0,1]: w, p w^{\prime} \in A C[0,1] \text { with } w \in(S L)_{0} \text { or }(N)_{0} \text { or }(P) \text { or }(B r)_{0}\right\}
$$

and notice that

$$
L: D(L)\left(\subseteq C[0,1] \subseteq L_{p q}^{2}[0,1]\right) \rightarrow L_{p q}^{1}[0,1]
$$

Theorem 4.1: Let $u_{n}$ be a sequence in $D(L)$. Suppose $u \in C[0,1]$ and $y \in L_{p q}^{1}[0,1]$ be such that $u_{n} \rightarrow u$ in $C[0,1]$ and $L u_{n} \rightarrow y$ in $L_{p q}^{1}[0,1]$. Thus $u \in D(L)$ and $L u=y$ a.e. on $[0,1]$.

Proof: Let $\|\cdot\|_{L^{1}},\|\cdot\|_{L_{p q}^{1}}$ and $|\cdot|_{0}$ denote the usual norms in $L^{1}[0,1], L_{p q}^{1}[0,1]$ and $C[0,1]$ respectively. For $n=1,2, \ldots$ there exist constants $C_{1}$ and $C_{2}$ independent of $n$ with

$$
\left\|\left(p u_{n}^{\prime}\right)^{\prime}+p r u_{n}\right\|_{L^{1}}=\left\|L u_{n}\right\|_{L_{p q}^{1}} \leq C_{1} \text { and }\left\|\left(p u_{n}^{\prime}\right)^{\prime}\right\|_{L^{1} \leq C_{2}}
$$

This together with the boundary condition implies that there exists a constant $C_{3}$ independent of $n$ with

$$
\left|p u_{n}^{\prime}\right|_{0} \leq C_{3}
$$

Consequently Sobolev's imbedding theorem [1] guarantees the existence of a subsequence $S$ of integers with $p u_{n}^{\prime} \rightarrow p u^{\prime}$ in $C[0,1]$ as $n \rightarrow \infty$ in $S$. For $x \in(0,1)$ and $n \in S$ we have

$$
p(x) u_{n}^{\prime}(x)=\lim _{t \rightarrow 0^{+}} p(t) u_{n}^{\prime}(t)+\int_{0}^{x}\left(p(s) u_{n}^{\prime}(s)^{\prime} d s\right.
$$

and this together with the fact that $\left(p u_{n}^{\prime}\right)^{\prime}+p r u_{n} \rightarrow-p q y$ in $L^{1}[0,1]$ implies

$$
p(x) u_{n}^{\prime}(x)=\lim _{t \rightarrow 0^{+}} p(t) u_{n}^{\prime}(t)+\int_{0}^{x} p(s)[-q(s) y(s)-r(s) u(s)] d s .
$$

Thus $u \in D(L)$ and $\left(p u^{\prime}\right)^{\prime}+p r u=-p q y$ a.e. on $[0,1]$.

Theorem 4.2: The eigenvalues, $\lambda$, of the eigenvalue problem (4.1) are real and the eigenfunctions corresponding to the distinct eigenvalues of (4.1) are orthogonal in $L_{p q}^{2}[0,1]$. In addition, the eigenvalues form at most a countable set with no finite limit point.

Proof: Suppose $\lambda_{0} \in \mathbf{R}$ is a limit point of the set of eigenvalues of (4.1). Then there exists a distinct sequence $\left\{\lambda_{n}\right\}, \lambda_{n} \neq \lambda_{0}$, of eigenvalues of (4.1) with $\lambda_{n} \rightarrow \lambda_{0}$. Let $\phi_{n}$ denote the eigenfunction for (4.1) corresponding to $\lambda_{n}$ and with $\left|\phi_{n}\right|_{0}=1$ for all $n$. Now $\phi_{n}$ satisfies the boundary condition and of course $L \phi_{n}=\lambda_{n} \phi_{n}$ a.e. on $[0,1]$. Thus

$$
\lambda_{n} \int_{0}^{1} p q\left|\phi_{n}\right|^{2} d t=A_{0}+\int_{0}^{1} p\left|\phi_{n}^{\prime}\right|^{2} d t-\int_{0}^{1} p r\left|\phi_{n}\right|^{2} d t
$$


where

$$
A_{0}=\left\{\begin{array}{l}
\frac{a}{b}\left|\phi_{n}(1)\right|^{2}+\frac{\alpha}{\beta}\left|\phi_{n}(0)\right|^{2}, \phi_{n} \in(S L)_{0} \\
0, \phi_{n} \in(N)_{0} \text { or }(P) \\
\frac{-\left|\phi_{n}(1)\right|^{2}}{\int_{0}^{1} \frac{d s}{p(s)}}, \phi_{n} \in(B r)_{0} .
\end{array}\right.
$$

This together with $\left|\phi_{n}\right|_{0}=1$ implies that there is a constant $C_{4}$ independent of $n$ with

$$
\int_{0}^{1} p\left|\phi_{n}^{\prime}\right|^{2} d t \leq C_{4}
$$

Consequently, $\left\{\phi_{n}\right\}$ is bounded and equicontinuous on $[0,1]$ so there exists a $\phi \in C[0,1]$ and a subsequence $S$ of integers with $\phi_{n} \rightarrow \phi$ in $C[0,1]$ as $n \rightarrow \infty$ in $S$. Let $n \in S$ and notice $L \phi_{n}=\lambda_{n} \phi_{n}$ a.e. on $[0,1]$ implies $L \phi_{n} \rightarrow \lambda_{0} \phi$ in $L_{p q}^{1}[0,1]$. Then theorem 4.1 implies $\phi \in D(L)$ and $L \phi=\lambda_{0} \phi$ a.e. on $[0,1]$. Notice also $|\phi|_{0}=1$ and so $\lambda_{0}$ is an eigenvalue of $L$. Now

$$
\left\langle\phi_{n}, \phi\right\rangle=\int_{0}^{1} p q \phi_{n} \bar{\phi} d t=0 \text { for all } n \in S
$$

so $\int_{0}^{1} p q|\phi|^{2} d t=0$. Consequently, $\phi(x)=0$ a.e. on $[0,1]$, a contradiction.

Now theorem 4.2 implies that there exists $\lambda^{*} \in \mathbf{R}$ such that $\lambda^{*}$ is not an eigenvalue of (4.1). Assume without loss of generality for the remainder of this section that 0 is not an eigenvalue of (4.1).

Let $y_{1}$ and $y_{2}$ be two linearly independent solutions of $\left(p y^{\prime}\right)^{\prime}+r p y=0$ a.e. on $[0,1]$ with $y_{1}, y_{2} \in C[0,1]$ and $p y_{1}^{\prime}, p y_{2}^{\prime} \in A C[0,1]$.

Remark: If $(S L)_{0}$ or $(N)_{0}$ is considered, choose $y_{2}$ as in theorem 2.2. For $(P)$ choose $y_{2}$ as in theorem 2.3 whereas for $(B r)_{0}$ choose $y_{2}$ as in theorem 2.4 .

Now for any $h \in L_{p q}^{1}[0,1]$ the boundary value problem

$$
\left\{\begin{array}{l}
L u=h \text { a.e. on }[0,1] \\
u \in(S L)_{0} \text { or }(N)_{0} \text { or }(P) \text { or }(B r)_{0}
\end{array}\right.
$$

has a unique solution

$$
L^{-1} h(t)=u(t)=A_{h} y_{1}(t)+B_{h} y_{2}(t)+\int_{0}^{t} \frac{\left[y_{1}(s) y_{2}(t)-y_{1}(t) y_{2}(s)\right]}{W(s)} q(s) h(s) d s
$$

where $A_{h}$ and $B_{h}$ may be constructed as in theorems $2.2,2.3$ or 2.4 ; see $[14,16]$. It follows 
immediately that

$$
L^{-1}: L_{p q}^{1}[0,1] \rightarrow D(L) \subseteq C[0,1] \subseteq L_{p q}^{2}[0,1] .
$$

The Arzela-Ascoli theorem (see $[14,16]$ or the ideas in theorem 2.2) implies that $L^{-1}$ is completely continuous. Next, define the imbedding $j: L_{p q}^{2}[0,1] \rightarrow L_{p q}^{1}[0,1]$ by $j u=u$. Note $j$ is continuous since Hölder's inequality yields

$$
\int_{0}^{1} p q|u| d t \leq\left(\int_{0}^{1} p q|u|^{2} d t\right)^{\frac{1}{2}}\left(\int_{0}^{1} p q d t\right)^{\frac{1}{2}}
$$

Consequently,

$$
L^{-1} j: L_{p q}^{2}[0,1] \rightarrow D(L) \subseteq L_{p q}^{2}[0,1]
$$

is completely continuous. In addition, $[14,16]$, for $u, v \in L_{p q}^{2}[0,1]$ it is easy to check that

$$
\left\langle L^{-1} j u, v\right\rangle=\left\langle u, L^{-1} j v\right\rangle \text {. }
$$

The spectral theorem for compact self adjoint operators [18] implies that $L$ has an countably infinite number of real eigenvalues $\lambda_{i}$ with corresponding eigenfunctions $u_{i} \in D(L)$. Of course

where

$$
\lambda_{i}=\frac{A_{0}+\int_{0}^{1} p\left|u_{i}^{\prime}\right|^{2} d t-\int_{0}^{1} p r\left|u_{i}\right|^{2} d t}{\int_{0}^{1} p q\left|u_{i}\right|^{2} d t}
$$

$$
A_{0}=\left\{\begin{array}{l}
\frac{a}{b}\left|u_{i}(1)\right|^{2}+\frac{\alpha}{\beta}\left|u_{i}(0)\right|^{2}, u_{i} \in(S L)_{0} \\
0, u_{i} \in(N)_{0} \text { or }(P) \\
\frac{-\left|u_{i}(1)\right|^{2}}{\int_{0}^{1} \frac{d s}{p(s)}}, u_{i} \in(B r)_{0} .
\end{array}\right.
$$

Remark: Notice that $\lambda_{i} \geq \frac{-\int_{0}^{1} p r\left|u_{i}\right|^{2} d t}{\int_{0}^{1} p q\left|u_{i}\right|^{2} d t}$. This is clear in all cases except maybe when $u_{i} \in(B r)_{0}$. However if $u_{i} \in(B r)_{0}$, then

$$
u_{i}(1)=\int_{0}^{1} \sqrt{p(s)} u_{i}^{\prime}(s) \frac{1}{\sqrt{p(s)}} d s \leq\left(\int_{0}^{1} p(s)\left|u_{i}^{\prime}(s)\right|^{2} d s\right)^{\frac{1}{2}}\left(\int_{0}^{1} \frac{d s}{p(s)}\right)^{\frac{1}{2}}
$$

and the result follows.

Now the eigenfunctions $u_{i}$ may be chosen so that they form a orthonormal set. We may also 
arrange the eigenvalues so that

$$
\lambda_{0}<\lambda_{1}<\lambda_{2}<\ldots
$$

In addition, [18, pp. 282, 373] implies that the set of eigenfunctions $u_{i}$ form a basis for $L_{p q}^{2}[0,1]$ and if $h \in L_{p q}^{2}[0,1]$ then $h$ has a Fourier series representation and $h$ satisfies Parseval's equality, i.e.,

$$
h=\sum_{i=0}^{\infty}\left\langle h, u_{i}\right\rangle u_{i} \text { and } \int_{0}^{1} p q|h|^{2} d t=\sum_{i=0}^{\infty}\left|\left\langle h, u_{i}\right\rangle\right|^{2} .
$$

\section{References}

[1] Adams, R., Sobolev Spaces, Academic Press, New York 1975.

[2] Atkinson, F.V., Discrete and Continuous Boundary Problems, Academic Press, New York 1964.

[3] Atkinson, F.V., Everitt, W.N., and Zettl, A., Regularization of a Sturm-Liouville problem with an interior singularity using quasi-derivatives, Diff. Int. Eq. 1 (1988), 213-221.

[4] Everitt, W.N., On the transformation theory of ordinary second order linear symmetric differential expressions, Czech. Math. J. 32 (1992), 275-306.

[5] Everitt, W.N. and Zettl, A., Generalized symmetric ordinary differential equations I: The general theory, Nieuw Archief Voor Wiskunde 27 (1979), 363-397.

[6] Everitt, W.N., Kwong, M.K., and Zettl, A., Oscillations of eigenfunctions of weighted regular Sturm Liouville problems, J. London Math. Soc. 27 (1983), 106-120.

[7] Fonda, A. and Mawhin, J., Quadratic forms, weighted eigenfunctions and boundary value problems for nonlinear second order ordinary differential equations, Proc. Royal Soc. Edinburgh 112A (1989), 145-153.

[8] Dugundji, J. and Granas, A., Fixed Point Theory, Monografie Matematyczne, PWN, War saw 1982.

[9] Granas, A., Guenther, R.B., and Lee, J.W., Some general existence principles in the Cara théodory theory of nonlinear systems, J. Math. Pures Appl. 70 (1990), 153-196.

[10] Gupta, C.P. and Mawhin, J., Weighted eigenvalue, eigenfunctions and boundary value problems for fourth order ordinary differential equations, Recent Trends in Differential Equations (edited by R.P. Agarwal), World Scientific Series in Appl. Anal. 1 (1992), 253267.

[11] Mawhin, J. and Omano, W., Two point boundary value problems for nonlinear perturb ations of some singular linear differential equations at resonance, Comm. Math. Univ. Carolinae 30 (1989), 537-550.

[12] Naimark, M.A., Linear Differential Operators Part II, Ungar Publ. Co., London 1968.

[13] Omari, P. and Zanolin, F., Nonresonance conditions on the potential for a second order periodic boundary value problem, Proc. Amer. Math. Soc. 117 (1993), 125-135.

[14] O'Regan, D., Solvability of some two point boundary value problems of Dirichlet, Neumann, or Periodic type, Dynamical Systems and Appl. 2 (1993), 163-182.

[15] O'Regan, D., Nonresonance and existence for singular boundary value problems, Nonlinear Analysis, to appear.

[16] O'Regan, D., Solvability to some second order boundary value problems using eigenvalues of Sturm Liouville problems, to appear.

[17] Powers, D., Boundary Value Problems, Harcourt Brace Jovanovich Publ., San Diego 1987.

[18] Stakgold, I., Green's Functions and Boundary Value Problems, John Wiley \& Sons, New York 1979. 


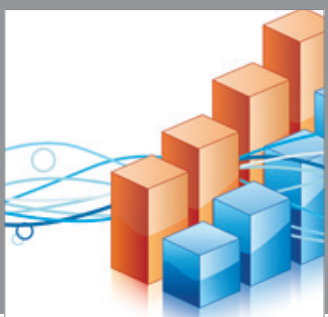

Advances in

Operations Research

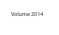

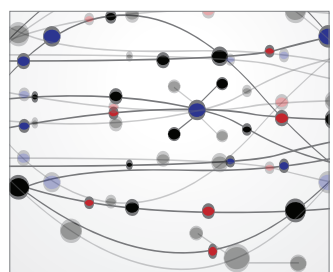

\section{The Scientific} World Journal
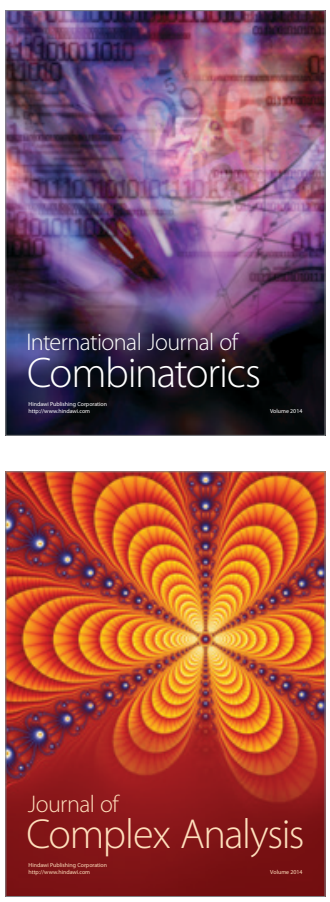

International Journal of

Mathematics and

Mathematical

Sciences
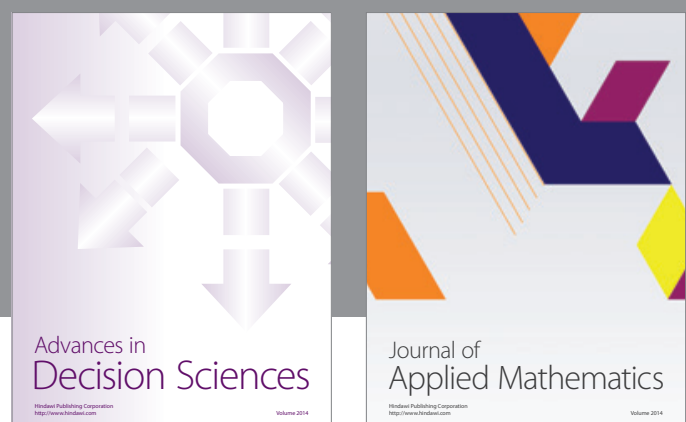

Journal of

Applied Mathematics
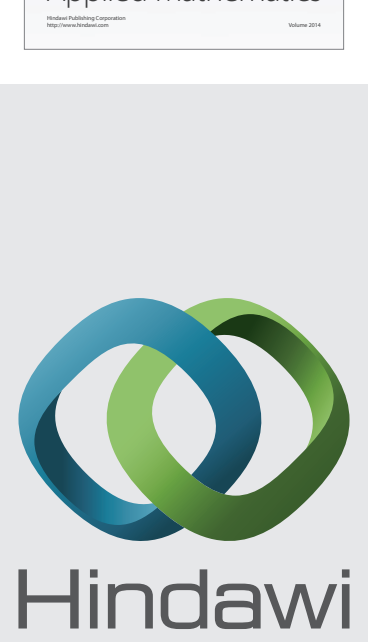

Submit your manuscripts at http://www.hindawi.com
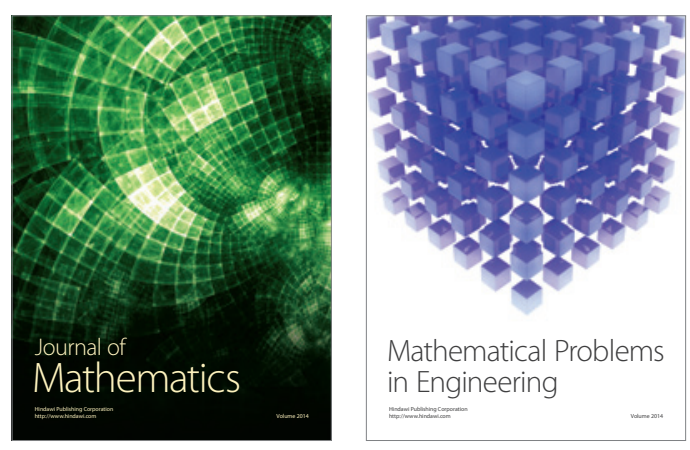

Mathematical Problems in Engineering
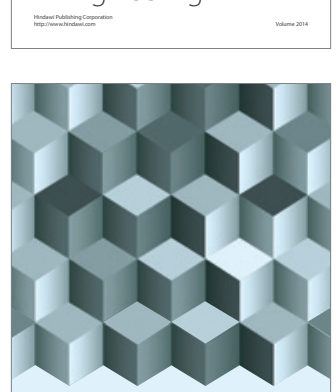

Journal of

Function Spaces
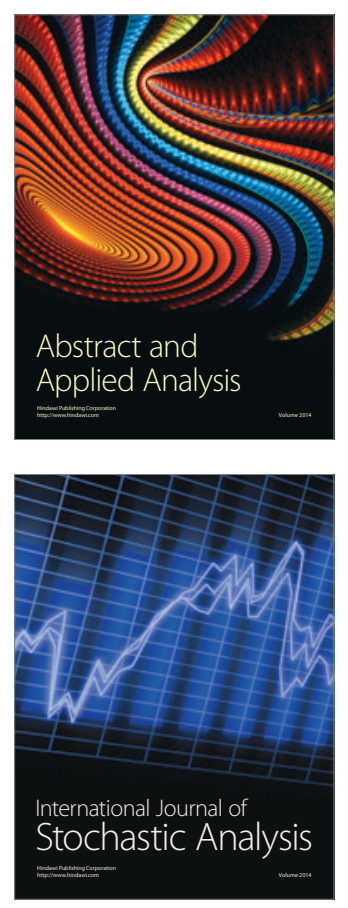

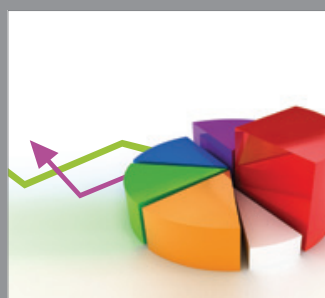

ournal of

Probability and Statistics

Promensencen
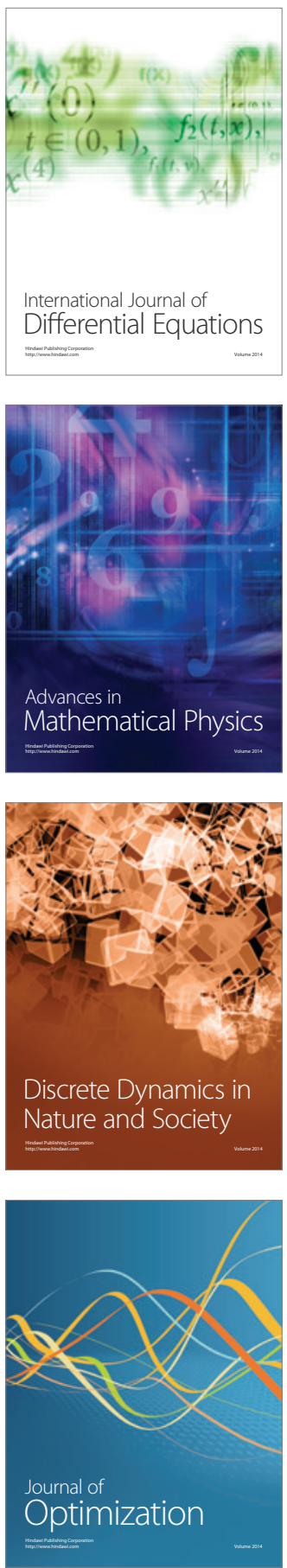